\title{
WEED INCIDENCE IN AN INTERCROPPING SYSTEM OF BANANA, TYPE PLANTAIN, CV. D'ANGOLA, WITH ASSAI PALM IN DIFFERENT ARRANGEMENTS
}

Ueliton Oliveira de Almeida ${ }^{1}$; Romeu de Carvalho Andrade Neto ${ }^{2}$; Aureny Maria Pereira Lunz ${ }^{2}$; Sebastião Elviro de Araújo Neto ${ }^{1}$; Leonardo Barreto Tavella ${ }^{1}$

\footnotetext{
${ }^{1}$ Universidade Federal do Acre. E-mail: uelitonhonda5@hotmail.com; selviro2000@yahoo.com.br; leo_tavella@hotmail.com

${ }^{2}$ EMBRAPA Acre. E-mail: romeu.andrade@embrapa.br; aureny.lunz@embrapa.br
}

\section{ABSTRACT}

The present study aimed at surveying weed species in the banana tree (Musa sp.) intercropping with assai (Euterpe precatoria). The experiment was performed using a randomized complete block design with six treatments (monocultures and intercropping) and four replications. The weed species were identified and quantified, enabling the calculation of phytosociological parameters. The soil cover and the dry mass of the weeds were evaluated. In general, Mollugo verticillata is one of the main species found in the essay. The E. precatoria palm monoculture showed a greater diversity of weed species and individuals and dry mass of the aerial part. Areas with banana monoculture or under a banana intercropping with E. precatoria palm in different planting arrangements displayed the same amount of dry matter of the aerial part and similar soil cover levels.

Keywords: Phytosociological survey, Musa sp., Euterpe precatoria

\section{OCORRÊNCIA DE PLANTAS DANINHAS NO CONSÓRCIO DE BANANEIRA, CV. D’ANGOLA, COM AÇAIZEIRO EM DIFERENTES ARRANJOS}

\section{RESUMO}

O presente estudo objetivou levantar espécies de plantas daninhas na bananeira (Musa sp.) consorciada com açaizeiro (Euterpe precatoria). O experimento foi conduzido em delineamento em blocos ao acaso, com seis tratamentos (monocultivos e consórcios) e quatro repetições. As espécies de plantas daninhas foram identificadas e quantificadas, possibilitando o cálculo de parâmetros fitossociológicos. A cobertura do solo e a massa seca das plantas daninhas foram 64 


\section{WEED INCIDENCE IN AN INTERCROPPING SYSTEM OF BANANA, TYPE PLANTAIN, CV.}

D'ANGOLA, WITH ASSAI PALM IN DIFFERENT ARRANGEMENTS

avaliadas. Em geral, Mollugo verticillata foi uma das principais espécies encontradas no ensaio. A monocultura de E. precatoria apresentou maior diversidade de espécies de plantas daninhas e indivíduos, assim como maior massa seca da parte aérea. Áreas com monocultivo de bananeira ou consorciada com $E$. precatoria em diferentes arranjos de plantio apresentaram a mesma quantidade de matéria seca da parte aérea de plantas daninhas e níveis de cobertura do solo semelhantes.

Palavras-chave: Levantamento fitossociológico, Musa sp., Euterpe precatoria

\section{INTRODUCTION}

Banana is the primary fruit species being produced in the State of Acre, with 102.949 tons for a harvested area of 8.118 ha, while Euterpe precatoria Mart. has an estimated production of 3.564 tons, equivalent to $0,8 \%$ of the national total (IBGE, 2019). Plantain tree (Musa sp.) and assai palm (E. precatoria) are strategic fruit plants for intercropping systems in the Amazon region, as the environmental conditions are favorable for crop growth.

One of the critical issues affecting plant cultivation, whether in monoculture, intercropping or agroforestry systems, is the negative interference of weed species (LANZA et al., 2017). These plants undermine agricultural profitability as they compete for resources that are essential to agricultural crops such as water, light, nutrients, space, and $\mathrm{CO} 2$, in addition to releasing allelopathic substances and acting as alternative hosts for plagues and diseases (MOURA FILHO et al., 2015). Further, when the intercrop provides a good soil cover, soil temperature will stay relatively low. This prevents burning of the organic matter in the soil and loss of nutrients. It also provides a microclimate that can be favourable for associated crops (OUMA; JERUTO, 2010).

The intercropping system of fruit tree species with other crops, whether annual, semiperennial or perennial, is a good alternative to optimize land use and an important approach to recover degraded areas. Consequently, it is possible to obtain a more efficient control of weeds due to a better area occupation and shading (SILVA et al., 2013). After studying the effect of banana plant densities "BRS Princess" on weeds suppression, Lanza et al. (2017) concluded that the results of their study point out advantages in relation to crop densities, increasing banana yield per equivalent area and, consequently, optimizing land use. These benefits also add to the ability to promote the suppression of common weeds in tropical regions.

In order to adopt the best weed management system in a crop, it is necessary to identify the 
species present in the area and classify them in terms of aggressiveness and class, taking into account primarily the phytosociological parameters, which are essential for the decision making regarding weed management and control (GOMES et al., 2010; OLIVEIRA; FREITAS, 2008; SOUZA et al., 2003), aiming at efficiency and economy.

The first stage of proper plant management in a crop involves the identification of the species present in the area, as well as those which are of greater importance, for that matter, are frequency, density and abundance. For this, phytosociological studies are carried out comparing the populations of weeds at a given time. Programmed repetitions of phytosociological studies may indicate trends in variation of the importance of one or more populations, and such variations may be associated with the agricultural practices adopted (SARMENTO et al., 2015), such as weed management, spacing, planting times, and cultural practices.

The objective of this work was to evaluate the occurrence of weeds in E. precatoria and plantain tree (Musa sp.), D'angola cultivar, in monoculture and intercropping systems in different planting arrangements.

\section{MATERIALS AND METHODS}

The study was carried out at the experimental field of Embrapa Acre, located in the municipality of Rio Branco, Acre, Brazil, at 1001'30" S, 67²42'18" W, and at an altitude of $160 \mathrm{~m}$. The regional climate is classified as Awi (hot and humid), according to Köppen, with maximum temperatures of $30.9^{\circ} \mathrm{C}$ and minimum of $20.8^{\circ} \mathrm{C}$, an average of $1648.9 \mathrm{~mm}$ of annual rainfall and relative humidity of $83 \%$. The survey was carried out on March 2014 in an experimental intercropping system of plantain tree, D’angola cv. (Musa sp., AAB - Terra subgroup) and single assai palm (Euterpe precatoria) in different planting arrangements.

The local soil is classified as dystrophic Red-Yellow Argisol, medium textured and welldrained, with the following physical-chemical characteristics in the 0-20 cm depth layer: $\mathrm{pH}\left(\mathrm{H}_{2} \mathrm{O}\right)$ $=4.52 ; \mathrm{Ca}=1.4 \mathrm{cmol}_{\mathrm{c}} \mathrm{dm}^{-3} ; \mathrm{Mg}=0.79 \mathrm{cmol}_{\mathrm{c}} \mathrm{dm}^{-3} ; \mathrm{K}=0.32 \mathrm{cmol}_{\mathrm{c}} \mathrm{dm}^{-3} ; \mathrm{Al}+\mathrm{H}=4.43 \mathrm{cmol}_{\mathrm{c}} \mathrm{dm}^{-}$ 3; $\mathrm{P}$ (surplus) $=9.91 \mathrm{mg} \mathrm{L}^{-1}$; base saturation $=36.18 \%$; sand $=315.42 \mathrm{~g} \mathrm{~kg}^{-1}$; silt $=319.88 \mathrm{~g} \mathrm{~kg}^{-1}$; clay $=364.70 \mathrm{~g} \mathrm{~kg}^{-1}$.

The experiment was performed using a randomized complete block design with six treatments and four replications, covering a total area of $5200 \mathrm{~m}^{2}$. The treatments consisted of crop systems, arranged as follows: Area 1 - E. precatoria monoculture in 4 x 3 m; Area 2 - Banana tree 


\section{WEED INCIDENCE IN AN INTERCROPPING SYSTEM OF BANANA, TYPE PLANTAIN, CV.}

D'ANGOLA, WITH ASSAI PALM IN DIFFERENT ARRANGEMENTS

monoculture in $3 \times 3 \mathrm{~m}$; Area 3 - Banana tree in $3 \times 2 \mathrm{~m}$ intercropped with E. precatoria in $3 \times 4$ $\mathrm{m}$; Area 4 - Banana tree in $3 \times 3 \mathrm{~m}$ intercropped with $E$. precatoria in $3 \times 4 \mathrm{~m}$; Area 5 - Banana tree in $4 \times 2 \times 2$ m intercropped with E. precatoria in $6 \times 3 \mathrm{~m}$; Area 6 - Banana tree in $4 \times 2 \times 2 \mathrm{~m}$ intercropped with E. precatoria in $4 \times 2 \times 3 \mathrm{~m}$.

The soil preparation was performed in a conventional manner, with one plowing and two harrowing operations. The planting holes, with dimensions of 0.4 x $0.4 \times 0.4 \mathrm{~m}$, were dug using a digger coupled to the tractor, 30 days before planting. To prepare the planting holes, $5 \mathrm{~kg}$ of chicken manure, $600 \mathrm{~g}$ of 10-10-10 (NPK) fertilizer, 800g of dolomitic limestone and 50g of micronutrients (FTE BR 12) were applied.

In March 2013, the banana trees were planted using seedlings composed of pieces of rhizomes, with a mass of approximately $400 \mathrm{~g}( \pm 50 \mathrm{~g})$, previously treated with carbofuran syrup, to prevent the attack of Cosmopolites sordidus Germar. The E. precatoria seedlings were planted in November 2013, when the banana trees had an average height of $1.6 \mathrm{~m}$, pseudo-stem perimeter at a height of $0.3 \mathrm{~m}$ from the soil of $0.4 \mathrm{~m}$ and 17 active leaves.

Topdressing fertilization was performed according to the technical recommendation for each crop, based on soil analysis. The banana plants were grown in a "mother, daughter and granddaughter" system, and the desiccation and defoliation were carried out when necessary. Weed management was initially performed using manual hoeing and later, with coastal brush cutter, every 30 days in the rainy season and 45-60 days in the dry season. Cultivation was conducted without irrigation.

To map out the weeds, a 0.25 per $\mathrm{m}^{2}(0.5 \times 0.5 \mathrm{~m})$ cast square was used and thrown lengthways three times, and thus it was placed to the right, at the center and to the left end of each plot. During the weed community sampling, the banana trees were in full production, with an average height of $3.20 \mathrm{~m}$, and the E. precatoria palms had an average height of $0.50 \mathrm{~m}$, measured from the soil up to the youngest expanded leaf.

After defining the sampling point of each square, which was performed by three trained evaluators through visual evaluation, the percentage of soil cover by weeds was estimated (Table $1)$.

Subsequently, the weed community was identified in relation to family, species and common name through comparisons to photos or descriptions of existing named specimens in the specialized literature (KISSMANN; GROTH, 1997; LORENZI, 2008). Then, the plants of each 
species were quantified separately, cut at ground level and stored in paper bags for drying in a forced air circulation oven at $60^{\circ} \mathrm{C}$ until a constant mass was reached. Afterwards, the material was weighed using an analytical balance $(0.01 \mathrm{~g})$ and the dry mass of each sample was converted into $\mathrm{kg} \mathrm{ha}^{-1}$.

Table 1 - Scale of visual assessment of soil cover by weeds. Rio Branco-AC, 2014.

\begin{tabular}{lll}
\hline Grade & Soil cover $(\%)$ & Level of cover \\
\hline 1 & 1.0 & Lack of plants \\
2 & $1.0-3.5$ & Low incidence \\
3 & $3.5-7.0$ & Reasonable incidence \\
4 & $7.0-12.5$ & Average incidence \\
5 & $12.5-20.0$ & Averagely high incidence \\
6 & $20.0-30.0$ & High incidence \\
7 & $30.0-50.0$ & Very high incidence \\
8 & $50.0-90.0$ & Extremely high incidence \\
9 & 100.0 & Total cover \\
\hline
\end{tabular}

Extracted and adapted from Deuber (1992).

Determination and counting of the species at each sampling point allowed the calculation of the following phytosociological variables: absolute density (D), relative density (Dr), absolute abundance (A), relative abundance (Ar), absolute frequency (F), relative frequency (Fr), importance value index (IVI), and relative importance (Ir), according to the procedure described by Carvalho \& Pitelli, 1992; Gomes et al., 2010; Silva et al., 2013.

The similarity index was calculated using the equation: $\mathrm{IS}=[2 . \mathrm{A} \div(\mathrm{B}+\mathrm{C}) \times 100]$, where $\mathrm{A}=$ number of species common to both areas; $\mathrm{B}=$ number of species from environment " $\mathrm{B}$ "; $\mathrm{C}=$ number of species from environment "C" (GOMES et al., 2010). Is ranges from 0 to 100\%, reaching the maximum level when all species are common to both areas and the minimum level when there are none in common.

Aerial dry matter mass (MSPA) and soil cover grades (NCS) were transformed by $\log _{\mathrm{x}}$ and, subsequently subjected to analysis of variance by the F test. Means were compared by the Tukey's test $(\mathrm{p} \leq 0.05)$. 


\section{WEED INCIDENCE IN AN INTERCROPPING SYSTEM OF BANANA, TYPE PLANTAIN, CV.}

D'ANGOLA, WITH ASSAI PALM IN DIFFERENT ARRANGEMENTS

\section{RESULTS AND DISCUSSION}

In the surveys carried out on E. precatoria and plantain monocultures, as well as in the intercropping areas with these fruits in different planting arrangements, 50 species, belonging to 17 families were identified (Table 2).

The families with the highest number of species were Poaceae (13), Cyperaceae (9) and Asteraceae (6), making up a total of $56 \%$ of the species identified. Of these species, $22 \%$ were common to all evaluated areas, regardless of the cropping system.

Regarding the classes, only areas 2 and 4 had a higher number of dicotyledonous species, with $55.17 \%$ and $59.26 \%$, respectively (Table 2). In some phytosociological surveys carried out on banana cropping, it was observed that dicotyledonous species prevail in the culture (GOMES et al., 2010; LIMA et al., 2012).

However, the weed community can be altered according to the farming systems management and spacings (BELALCÁZAR CARJAVAL et al., 1991; ERASMO et al., 2004), a factor that probably allowed a balance in the other areas, with 50\% monocotyledons and 50\% of dicotyledons. In the E. precatoria palm monoculture (area 1), the diversity and weed quantity was superior to the other areas, with 2333 individuals distributed among 34 species (Table 3).

It can be observed that Digitaria sanguinalis and Mollugo verticillata species were predominant, with an absolute frequency of 0.8 ; density of 413.0 and 205.3 plants per $\mathrm{m}^{2}$; absolute abundance of 123.9 and 61.6; relative frequency of 9.2\%; relative abundance of 35.9 and $17.9 \%$; relative density of 53.1 and $26.4 \%$; relative importance of 32.7 and $17.8 \%$, and; importance value index of 98.2 and 53.4, respectively (Table 3). In cassava growing areas, Albuquerque et al. (2014) found that Digitaria sanguinalis was the main species, showing higher density and abundance values, similar to the crops observed in the present study.

Wider spacings and the low height of E. precatoria palms when the survey was performed favored a higher lighting level on the weeds in the monoculture, increasing the number of individuals of Digitaria sanguinalis. This species performs C4 photosynthesis, which under conditions of high light intensity may represent a strong competitive advantage in relation to C3 plants, due to a greater carboxylation efficiency (LARCHER, 2004), standing out in the evaluated phytosociological parameters. The high reproductive capacity of this species may also have influenced the relative density $(53.11 \%)$, as it can produce up to 150,000 seeds per clump (LORENZI, 2008). 
Table 2 - Identification of weed communities in different cropping systems, according to family, species and common name. Rio Branco-AC, 2014.

\begin{tabular}{|c|c|c|c|c|c|c|c|}
\hline \multirow[t]{2}{*}{ Family/Species } & \multirow[t]{2}{*}{ Common name } & \multicolumn{6}{|c|}{ Cropping systems } \\
\hline & & 1 & 2 & 3 & 4 & 5 & 6 \\
\hline \multicolumn{8}{|l|}{ Amaranthaceae } \\
\hline Alternanthera tenella & basil & $\mathrm{x}$ & & & $\mathrm{x}$ & & \\
\hline \multicolumn{8}{|l|}{ Asteraceae } \\
\hline Ageratum conyzoides & billygoat weed & & $\mathrm{x}$ & & & & \\
\hline Corchorus olitorius & jute mallow & $\mathrm{x}$ & $\mathrm{x}$ & $\mathrm{x}$ & & $\mathrm{x}$ & $\mathrm{x}$ \\
\hline Emilia coccinea & tasselflower & $\mathrm{x}$ & & & & & \\
\hline Emilia fosbergii & florida tasselflower & & & & & $\mathrm{x}$ & \\
\hline Praxelis pauciflora & praxelis & $\mathrm{X}$ & $\mathrm{x}$ & $\mathrm{x}$ & $\mathrm{x}$ & $\mathrm{x}$ & $\mathrm{x}$ \\
\hline Vernonia polyanthes & ironweed & & & $\mathrm{x}$ & $\mathrm{x}$ & & \\
\hline \multicolumn{8}{|l|}{ Commelinaceae } \\
\hline Commelina benghalensis & benghal dayflower & $\mathrm{x}$ & & & $\mathrm{x}$ & $\mathrm{x}$ & $\mathrm{x}$ \\
\hline Murdannia nudiflora & dayflower & $\mathrm{x}$ & $\mathrm{x}$ & $\mathrm{x}$ & & $\mathrm{x}$ & $\mathrm{x}$ \\
\hline \multicolumn{8}{|l|}{ Convolvulaceae } \\
\hline Ipomoea triloba & littlebell & & $\mathrm{x}$ & & & & $\mathrm{x}$ \\
\hline \multicolumn{8}{|l|}{ Cyperaceae } \\
\hline Cyperus sp. & sedge & $\mathrm{x}$ & & & & & \\
\hline Cyperus difusus & diffused flatsedge & & & & & & $\mathrm{x}$ \\
\hline Cyperus esculentus & yellow nutsedge & $\mathrm{x}$ & $\mathrm{x}$ & $\mathrm{x}$ & $\mathrm{x}$ & $\mathrm{x}$ & $\mathrm{x}$ \\
\hline Cyperus flavus & yellow flatsedge & & & & & & $\mathrm{x}$ \\
\hline Cyperus iria & ricefield flatsedge & $\mathrm{x}$ & $\mathrm{x}$ & $\mathrm{x}$ & $\mathrm{x}$ & $\mathrm{x}$ & $\mathrm{x}$ \\
\hline Fimbristylis dichotoma & forked fimbry & $\mathrm{x}$ & $\mathrm{x}$ & $\mathrm{x}$ & $\mathrm{x}$ & $\mathrm{x}$ & $\mathrm{x}$ \\
\hline Kyllinga brevifolia & shortleaf spikesedge & & $\mathrm{x}$ & $\mathrm{x}$ & & $\mathrm{x}$ & \\
\hline Kyllinga odorata & fragrant spikesedge & $\mathrm{x}$ & $\mathrm{x}$ & $\mathrm{x}$ & $\mathrm{x}$ & $\mathrm{x}$ & $\mathrm{x}$ \\
\hline Rhychospora nervosa & beakrush & $\mathrm{x}$ & & $\mathrm{x}$ & & & \\
\hline \multicolumn{8}{|l|}{ Euphorbiaceae } \\
\hline Acalypha arvensis & field copperleaf & $\mathrm{x}$ & $\mathrm{x}$ & $\mathrm{x}$ & $\mathrm{x}$ & $\mathrm{x}$ & \\
\hline \multicolumn{8}{|l|}{ Fabaceae } \\
\hline Pueraria phaseoloides & tropical kudzu & $\mathrm{x}$ & $\mathrm{x}$ & & $\mathrm{x}$ & & \\
\hline Chamaecrista rotundifolia & roundleaf sensitive pea & & & & & & $\mathrm{x}$ \\
\hline Desmodium adscendens & zarzabacoa galana & $\mathrm{x}$ & $\mathrm{x}$ & & & & \\
\hline Desmodium barbatum & zarzabacoa peluda & & & & $\mathrm{x}$ & & \\
\hline \multicolumn{8}{|l|}{ Loganiaceae } \\
\hline Spigelia anthelmia & west Indian pinkroot & $\mathrm{x}$ & $\mathrm{x}$ & $\mathrm{x}$ & $\mathrm{x}$ & $\mathrm{x}$ & $\mathrm{x}$ \\
\hline \multicolumn{8}{|l|}{ Malvaceae } \\
\hline Malva spp. & mallow & $\mathrm{X}$ & & & & & \\
\hline Anoda cristata & crested anoda & $\mathrm{x}$ & & & & & \\
\hline Urena lobata & caesarweed & & $\mathrm{X}$ & & & & \\
\hline
\end{tabular}

Continue... 
Table 2 - Identification of weed communities in different cropping systems, according to family, species and common name. Rio Branco-AC, 2014.

\begin{tabular}{|c|c|c|c|c|c|c|c|}
\hline \multirow[t]{2}{*}{ Family/Species } & \multirow[t]{2}{*}{ Common name } & \multicolumn{6}{|c|}{ Cropping systems } \\
\hline & & 1 & 2 & 3 & 4 & 5 & 6 \\
\hline \multicolumn{8}{|l|}{ Molluginaceae } \\
\hline Mollugo verticillata & green carpetweed & $\mathrm{x}$ & $\mathrm{x}$ & $\mathrm{X}$ & $\mathrm{x}$ & $\mathrm{x}$ & $\mathrm{x}$ \\
\hline \multicolumn{8}{|l|}{ Phyllanthaceae } \\
\hline Phyllanthus niruri & gale of the wind & $\mathrm{X}$ & $\mathrm{x}$ & $\mathrm{x}$ & $\mathrm{x}$ & $\mathrm{x}$ & $\mathrm{x}$ \\
\hline Phyllanthus tenellus & mascarene island leaf-flower & $\mathrm{x}$ & & $\mathrm{X}$ & $\mathrm{x}$ & & $\mathrm{x}$ \\
\hline \multicolumn{8}{|l|}{ Piperaceae } \\
\hline Pothomorphe umbellata & monkey's hand & & & $\mathrm{X}$ & & & \\
\hline \multicolumn{8}{|l|}{ Plantaginaceae } \\
\hline Lindernia dubia & yellowseed false pimpernel & $\mathrm{x}$ & $\mathrm{x}$ & $\mathrm{X}$ & $\mathrm{x}$ & & \\
\hline Stemodia verticillata & whorled twintip & & $\mathrm{x}$ & & & & \\
\hline \multicolumn{8}{|l|}{ Poaceae } \\
\hline Brachiaria brizantha & palisade grass & $\mathrm{X}$ & $\mathrm{x}$ & & $\mathrm{x}$ & & \\
\hline Brachiaria decumbens & spreading liverseed grass & $\mathrm{x}$ & & & $\mathrm{x}$ & & \\
\hline Digitaria bicornis & asian crabgrass & $\mathrm{x}$ & & & & & \\
\hline Digitaria horizontalis & jamaican crabgrass & $\mathrm{x}$ & & & $\mathrm{x}$ & & \\
\hline Digitaria sanguinalis & hairy crabgrass & $\mathrm{x}$ & $\mathrm{x}$ & $\mathrm{x}$ & $\mathrm{x}$ & $\mathrm{x}$ & $\mathrm{x}$ \\
\hline Eleusine indica & indian goosegrass & $\mathrm{x}$ & $\mathrm{x}$ & $\mathrm{X}$ & & $\mathrm{x}$ & $\mathrm{x}$ \\
\hline Leptochloa filiformis & mucronate sprangeltop & $\mathrm{x}$ & $\mathrm{x}$ & $\mathrm{x}$ & $\mathrm{x}$ & $\mathrm{x}$ & \\
\hline Panicum maximum & guineagrass & $\mathrm{x}$ & & & & & \\
\hline Paspalum conjugatum & hilograss & $\mathrm{x}$ & & & $\mathrm{x}$ & $\mathrm{x}$ & $\mathrm{x}$ \\
\hline Paspalum maritimum & coastal sand paspalum & $\mathrm{x}$ & $\mathrm{x}$ & $\mathrm{X}$ & $\mathrm{x}$ & $\mathrm{x}$ & $\mathrm{x}$ \\
\hline Paspalum notatum & bahiagrass & $\mathrm{x}$ & $\mathrm{x}$ & $\mathrm{x}$ & $\mathrm{x}$ & & $\mathrm{x}$ \\
\hline Paspalum paniculatum & arrocillo & & $\mathrm{x}$ & $\mathrm{X}$ & & $\mathrm{x}$ & \\
\hline Sporobolus indicus & smut grass & & $\mathrm{x}$ & $\mathrm{X}$ & & & $\mathrm{X}$ \\
\hline \multicolumn{8}{|l|}{ Portulacaceae } \\
\hline Talinum paniculatum & jewels of opar & & & & $\mathrm{x}$ & $\mathrm{x}$ & \\
\hline \multicolumn{8}{|l|}{ Rubiaceae } \\
\hline Spermacoce latifolia & oval-leaf false buttonweed & $\mathrm{x}$ & $\mathrm{x}$ & $\mathrm{X}$ & $\mathrm{x}$ & $\mathrm{x}$ & $\mathrm{x}$ \\
\hline \multicolumn{8}{|l|}{ Urticaceae } \\
\hline Cecropia pachystachya & yarumo & & $\mathrm{x}$ & $\mathrm{X}$ & $\mathrm{X}$ & & \\
\hline
\end{tabular}


Table 3 - Values of the number of squares (NQ), number of individuals (NI), absolute frequency $(\mathrm{Fa})$, absolute density ( $\mathrm{Da})$, absolute abundance $(\mathrm{Ab})$, relative frequency $(\mathrm{Fr})$, relative abundance (Ar), relative density (Dr), relative importance (Ir) and importance value index (IVI) in area 1. Rio Branco-AC, 2014.

\begin{tabular}{|c|c|c|c|c|c|c|c|c|c|c|}
\hline Species & $\mathrm{NI}$ & NQ & $\mathrm{Fa}$ & $\begin{array}{l}\mathrm{Da} \\
\left(\mathrm{p} \mathrm{m}^{2}\right)\end{array}$ & $\mathrm{Ab}$ & Fr $(\%)$ & $\operatorname{Ar}(\%)$ & $\operatorname{Dr}(\%)$ & $\operatorname{Ir}(\%)$ & IVI \\
\hline Acalypha arvensis & 1 & 1 & 0.1 & 0.3 & 1.0 & 0.9 & 0.3 & 0.0 & 0.4 & 1.3 \\
\hline Alternanthera tenella & 4 & 2 & 0.2 & 1.3 & 2.0 & 1.8 & 0.6 & 0.2 & 0.9 & 2.6 \\
\hline Anoda cristata & 1 & 1 & 0.1 & 0.3 & 1.0 & 0.9 & 0.3 & 0.0 & 0.4 & 1.3 \\
\hline Brachiaria brizantha & 4 & 4 & 0.3 & 1.3 & 1.0 & 3.7 & 0.3 & 0.2 & 1.4 & 4.1 \\
\hline Brachiaria decumbens & 1 & 1 & 0.1 & 0.3 & 1.0 & 0.9 & 0.3 & 0.0 & 0.4 & 1.3 \\
\hline Commelina benghalensis & 2 & 2 & 0.2 & 0.7 & 1.0 & 1.8 & 0.3 & 0.1 & 0.7 & 2.2 \\
\hline Corchorus olitorius & 11 & 5 & 0.4 & 3.7 & 2.2 & 4.6 & 0.6 & 0.5 & 1.9 & 5.7 \\
\hline Cyperus esculentus & 5 & 3 & 0.3 & 1.7 & 1.7 & 2.8 & 0.5 & 0.2 & 1.2 & 3.5 \\
\hline Cyperus iria & 3 & 2 & 0.2 & 1.0 & 1.5 & 1.8 & 0.4 & 0.1 & 0.8 & 2.4 \\
\hline Cyperus spp. & 1 & 1 & 0.1 & 0.3 & 1.0 & 0.9 & 0.3 & 0.0 & 0.4 & 1.3 \\
\hline Desmodium adscendens & 1 & 1 & 0.1 & 0.3 & 1.0 & 0.9 & 0.3 & 0.0 & 0.4 & 1.3 \\
\hline Digitaria bicornis & 9 & 1 & 0.1 & 3.0 & 9.0 & 0.9 & 2.6 & 0.4 & 1.3 & 3.9 \\
\hline Digitaria horizontalis & 7 & 2 & 0.2 & 2.3 & 3.5 & 1.8 & 1.0 & 0.3 & 1.1 & 3.2 \\
\hline Digitaria sanguinalis & 1239 & 10 & 0.8 & 413.0 & 123.9 & 9.2 & 35.9 & 53.1 & 32.7 & 98.2 \\
\hline Eleusine indica & 20 & 3 & 0.3 & 6.7 & 6.7 & 2.8 & 1.9 & 0.9 & 1.8 & 5.5 \\
\hline Emilia coccinea & 11 & 2 & 0.2 & 3.7 & 5.5 & 1.8 & 1.6 & 0.5 & 1.3 & 3.9 \\
\hline Fimbristylis dichotoma & 18 & 5 & 0.4 & 6.0 & 3.6 & 4.6 & 1.0 & 0.8 & 2.1 & 6.4 \\
\hline Kyllinga odorata & 35 & 2 & 0.2 & 11.7 & 17.5 & 1.8 & 5.1 & 1.5 & 2.8 & 8.4 \\
\hline Leptochloa filiformis & 16 & 3 & 0.3 & 5.3 & 5.3 & 2.8 & 1.5 & 0.7 & 1.7 & 5.0 \\
\hline Lindernia dubia & 57 & 5 & 0.4 & 19.0 & 11.4 & 4.6 & 3.3 & 2.4 & 3.4 & 10.3 \\
\hline Malva spp. & 4 & 2 & 0.2 & 1.3 & 2.0 & 1.8 & 0.6 & 0.2 & 0.9 & 2.6 \\
\hline Mollugo verticillata & 616 & 10 & 0.8 & 205.3 & 61.6 & 9.2 & 17.9 & 26.4 & 17.8 & 53.4 \\
\hline Murdannia nudiflora & 94 & 7 & 0.6 & 31.3 & 13.4 & 6.4 & 3.9 & 4.0 & 4.8 & 14.3 \\
\hline Panicum maximum & 4 & 2 & 0.2 & 1.3 & 2.0 & 1.8 & 0.6 & 0.2 & 0.9 & 2.6 \\
\hline Paspalum conjugatum & 46 & 2 & 0.2 & 15.3 & 23.0 & 1.8 & 6.7 & 2.0 & 3.5 & 10.5 \\
\hline Paspalum maritimum & 46 & 5 & 0.4 & 15.3 & 9.2 & 4.6 & 2.7 & 2.0 & 3.1 & 9.2 \\
\hline Paspalum notatum & 1 & 1 & 0.1 & 0.3 & 1.0 & 0.9 & 0.3 & 0.0 & 0.4 & 1.3 \\
\hline Phyllanthus niruri & 15 & 5 & 0.4 & 5.0 & 3.0 & 4.6 & 0.9 & 0.6 & 2.0 & 6.1 \\
\hline Phyllanthus tenellus & 4 & 2 & 0.2 & 1.3 & 2.0 & 1.8 & 0.6 & 0.2 & 0.9 & 2.6 \\
\hline Praxelis pauciflora & 19 & 5 & 0.4 & 6.3 & 3.8 & 4.6 & 1.1 & 0.8 & 2.2 & 6.5 \\
\hline Pueraria phaseoloides & 9 & 4 & 0,3 & 3,0 & 2,3 & 3,7 & 0,7 & 0.4 & 1.6 & 4.7 \\
\hline Rhychospora nervosa & 17 & 1 & 0.1 & 5.7 & 17.0 & 0.9 & 4.9 & 0.7 & 2.2 & 6.6 \\
\hline Spermacoce latifolia & 7 & 5 & 0.4 & 2.3 & 1.4 & 4.6 & 0.4 & 0.3 & 1.8 & 5.3 \\
\hline Spigelia anthelmia & 5 & 2 & 0.2 & 1.7 & 2.5 & 1.8 & 0.7 & 0.2 & 0.9 & 2.8 \\
\hline Total & 2333 & - & 9.1 & 777.7 & 344.9 & 100 & 100 & 100 & 100 & - \\
\hline
\end{tabular}




\section{WEED INCIDENCE IN AN INTERCROPPING SYSTEM OF BANANA, TYPE PLANTAIN, CV.}

D'ANGOLA, WITH ASSAI PALM IN DIFFERENT ARRANGEMENTS

In the banana tree monoculture (area 2), 770 individuals were identified and grouped into 14 families and 28 species (Tables 2 and 4).

The species with the highest absolute and relative frequencies in descending order were Mollugo verticillata, Kyllinga odorata, Paspalum maritimum, and Digitaria sanguinalis. Among these species showing a high frequency, two belong to Poaceae family, which can be explained by the greater efficiency of the soil production factors. In a study carried out by Silva et al. (2013) with the objective of evaluating the occurrence of weeds in intercropped coffee and macadamia nuts, a higher occurrence of weeds of the family Poaceae.

In relation to the other parameters, Paspalum maritimum, Mollugo verticillata and Paspalum paniculatum displayed higher absolute density values, 60.3, 55.7 and 53.3 plants per $\mathrm{m}^{2}$; absolute abundance rates of 30.2, 23.9 and 53.3; relative abundance rates of 15.5, 12.3 and 27.5\%; relative density of 23.5, 21.7 and $20.8 \%$; relative importance of 15.6, 14.3 and $17.4 \%$, and; value index of importance of 46.7, 43.0 and 52.1; respectively. The tolerance to shaded and intense environments shown by some species of the genus Paspalum is confirmed in some studies, as it was observed in Paspalum reginelli, P. dilatatum, P. notatum (BARRO et al., 2010; SCHREINER, 1987), which may explain the predominance of these weeds.

In the $3 \times 2 \mathrm{~m}$ banana intercropped with $3 \times 4 \mathrm{~m}$ E. precatoria (area 3), Mollugo verticillata showed an absolute frequency of 0.7, followed by Paspalum maritimum and Spigelia anthelmia with a rate of 0.6 , and these were also the species displaying the highest relative frequency (Table $5)$.

Mollugo verticillata also obtained higher values for absolute density, with 126 plants per $\mathrm{m}^{2}$; absolute abundance of 47.3 ; relative abundance of $29 \%$; relative density of $57.4 \%$; relative importance of $32.7 \%$, and importance value index of 98.2. In Brazil, these species are characterized by infesting annual and perennial crops (LORENZI, 2008).

Table 6 also shows the survey carried out on weed found in the $3 \times 3 \mathrm{~m}$ banana intercropped with $3 \times 4 \mathrm{~m}$ E. precatoria palm (area 4), as well as the phytosociological parameters.

In descending order, the seven most frequent species in absolute and relative forms were: Mollugo verticillata with 0.9 and 13.4\%, Spigelia anthelmia and Praxelis pauciflora with 0.5 and 7.3\%, Spermacoce latifolia, Cyperus esculentus, Kyllinga odorata, and Paspalum maritimum with 0.4 and 6.1\%, respectively. However, Mollugo verticillata showed the highest absolute density 
(209 plants per $\mathrm{m}^{2}$ ), absolute abundance (57), relative abundance $(43.3 \%)$, relative density $(71.7 \%)$, relative importance (42.8\%), and importance value index (128.4).

Table 4 - Values of the number of individuals (NI), number of squares (NQ), absolute frequency $(\mathrm{Fa})$, absolute density $(\mathrm{Da})$, absolute abundance $(\mathrm{Ab})$, relative frequency $(\mathrm{Fr})$, relative abundance (Ar), relative density (Dr), relative importance (Ir) and the importance value index (IVI) in area 2. Rio Branco-AC, 2014.

\begin{tabular}{|c|c|c|c|c|c|c|c|c|c|c|}
\hline Species & NI & NQ & $\mathrm{Fa}$ & $\begin{array}{l}\mathrm{Da} \\
\left(\mathrm{p} \mathrm{m}^{2}\right)\end{array}$ & $\mathrm{Ab}$ & $\operatorname{Fr}(\%)$ & $\operatorname{Ar}(\%)$ & $\operatorname{Dr}(\%)$ & $\operatorname{Ir}(\%)$ & IVI \\
\hline Acalypha arvensis & 2 & 2 & 0.2 & 0.7 & 1.0 & 2.6 & 0.5 & 0.3 & 1.1 & 3.3 \\
\hline Agetarum conyzoides & 7 & 2 & 0.2 & 2.3 & 3.5 & 2.6 & 1.8 & 0.9 & 1.8 & 5.3 \\
\hline Brachiaria brizantha & 5 & 2 & 0.2 & 1.7 & 2.5 & 2.6 & 1.3 & 0.6 & 1.5 & 4.5 \\
\hline Cecropia pachystachya & 4 & 2 & 0.2 & 1.3 & 2.0 & 2.6 & 1.0 & 0.5 & 1.4 & 4.1 \\
\hline Corchorus olitorius & 1 & 1 & 0.1 & 0.3 & 1.0 & 1.3 & 0.5 & 0.1 & 0.6 & 1.9 \\
\hline Cyperus esculentus & 5 & 2 & 0.2 & 1.7 & 2.5 & 2.6 & 1.3 & 0.6 & 1.5 & 4.5 \\
\hline Cyperus iria & 20 & 2 & 0.2 & 6.7 & 10.0 & 2.6 & 5.2 & 2.6 & 3.4 & 10.3 \\
\hline Desmodium adscendens & 2 & 1 & 0.1 & 0.7 & 2.0 & 1.3 & 1.0 & 0.3 & 0.9 & 2.6 \\
\hline Digitaria sanguinalis & 40 & 5 & 0.4 & 13.3 & 8.0 & 6.4 & 4.1 & 5.2 & 5.2 & 15.7 \\
\hline Eleusine indica & 1 & 1 & 0.1 & 0.3 & 1.0 & 1.3 & 0.5 & 0.1 & 0.6 & 1.9 \\
\hline Fimbristylis dichotoma & 15 & 3 & 0.3 & 5.0 & 5.0 & 3.8 & 2.6 & 1.9 & 2.8 & 8.4 \\
\hline Ipomoea triloba & 2 & 2 & 0.2 & 0.7 & 1.0 & 2.6 & 0.5 & 0.3 & 1.1 & 3.3 \\
\hline Kyllinga brevifolia & 4 & 1 & 0.1 & 1.3 & 4.0 & 1.3 & 2.1 & 0.5 & 1.3 & 3.9 \\
\hline Kyllinga odorata & 72 & 7 & 0.6 & 24.0 & 10.3 & 9.0 & 5.3 & 9.4 & 7.9 & 23.6 \\
\hline Leptochloa filiformis & 14 & 3 & 0.3 & 4.7 & 4.7 & 3.8 & 2.4 & 1.8 & 2.7 & 8.1 \\
\hline Lindernia dubia & 8 & 4 & 0.3 & 2.7 & 2.0 & 5.1 & 1.0 & 1.0 & 2.4 & 7.2 \\
\hline Mollugo verticillata & 167 & 7 & 0.6 & 55.7 & 23.9 & 9.0 & 12.3 & 21.7 & 14.3 & 43.0 \\
\hline Murdannia nudiflora & 4 & 2 & 0.2 & 1.3 & 2.0 & 2.6 & 1.0 & 0.5 & 1.4 & 4.1 \\
\hline Paspalum maritimum & 181 & 6 & 0.5 & 60.3 & 30.2 & 7.7 & 15.5 & 23.5 & 15.6 & 46.7 \\
\hline Paspalum notatum & 8 & 2 & 0.2 & 2.7 & 4.0 & 2.6 & 2.1 & 1.0 & 1.9 & 5.7 \\
\hline Paspalum paniculatum & 160 & 3 & 0.3 & 53.3 & 53.3 & 3.8 & 27.5 & 20.8 & 17.4 & 52.1 \\
\hline Phyllanthus niruri & 10 & 4 & 0.3 & 3.3 & 2.5 & 5.1 & 1.3 & 1.3 & 2.6 & 7.7 \\
\hline Praxelis pauciflora & 10 & 3 & 0.3 & 3.3 & 3.3 & 3.8 & 1.7 & 1.3 & 2.3 & 6.9 \\
\hline Pueraria phaseoloides & 1 & 1 & 0.1 & 0.3 & 1.0 & 1.3 & 0.5 & 0.1 & 0.6 & 1.9 \\
\hline Spermacoce latifolia & 10 & 3 & 0.3 & 3.3 & 3.3 & 3.8 & 1.7 & 1.3 & 2.3 & 6.9 \\
\hline Spigelia anthelmia & 8 & 3 & 0.3 & 2.7 & 2.7 & 3.8 & 1.4 & 1.0 & 2.1 & 6.3 \\
\hline Sporobolus indicus & 2 & 1 & 0.1 & 0.7 & 2.0 & 1.3 & 1.0 & 0.3 & 0.9 & 2.6 \\
\hline Stemodia verticillata & 4 & 1 & 0.1 & 1.3 & 4.0 & 1.3 & 2.1 & 0.5 & 1.3 & 3.9 \\
\hline Urena lobata & 3 & 2 & 0.2 & 1.0 & 1.5 & 2.6 & 0.8 & 0.4 & 1.2 & 3.7 \\
\hline Total & 770 & - & 6.5 & 256.7 & 194.1 & 100 & 100 & 100 & 100 & - \\
\hline
\end{tabular}


Table 5 - Values of the number of individuals (NI), number of squares (NQ), absolute frequency $(\mathrm{Fa})$, absolute density $(\mathrm{Da})$, absolute abundance $(\mathrm{Ab})$, relative frequency $(\mathrm{Fr})$, relative abundance (Ar), relative density (Dr), relative importance (Ir) and the importance value index (IVI) in area 3. Rio Branco-AC, 2014.

\begin{tabular}{|c|c|c|c|c|c|c|c|c|c|c|}
\hline Species & NI & NQ & $\mathrm{Fa}$ & $\begin{array}{l}\mathrm{Da} \\
\left(\mathrm{p} \mathrm{m}^{2}\right)\end{array}$ & $\mathrm{Ab}$ & $\operatorname{Fr}(\%)$ & $\operatorname{Ar}(\%)$ & $\operatorname{Dr}(\%)$ & $\operatorname{Ir}(\%)$ & IVI \\
\hline Acalypha arvensis & 1 & 1 & 0.1 & 0.3 & 1.0 & 1.5 & 0.6 & 0.2 & 0.7 & 2.2 \\
\hline Cecropia pachystachya & 2 & 1 & 0.1 & 0.7 & 2.0 & 1.5 & 1.2 & 0.3 & 1.0 & 3.0 \\
\hline Corchorus olitorius & 1 & 1 & 0.1 & 0.3 & 1.0 & 1.5 & 0.6 & 0.2 & 0.7 & 2.2 \\
\hline Cyperus esculentus & 1 & 1 & 0.1 & 0.3 & 1.0 & 1.5 & 0.6 & 0.2 & 0.7 & 2.2 \\
\hline Cyperus iria & 3 & 1 & 0.1 & 1.0 & 3.0 & 1.5 & 1.8 & 0.5 & 1.3 & 3.8 \\
\hline Digitaria sanguinalis & 39 & 4 & 0.3 & 13.0 & 9.8 & 5.9 & 6.0 & 5.9 & 5.9 & 17.8 \\
\hline Eleusine indica & 2 & 2 & 0.2 & 0.7 & 1.0 & 2.9 & 0.6 & 0.3 & 1.3 & 3.9 \\
\hline Fimbristylis dichotoma & 19 & 3 & 0.3 & 6.3 & 6.3 & 4.4 & 3.9 & 2.9 & 3.7 & 11.2 \\
\hline Kyllinga brevifolia & 11 & 2 & 0.2 & 3.7 & 5.5 & 2.9 & 3.4 & 1.7 & 2.7 & 8.0 \\
\hline Kyllinga odorata & 25 & 4 & 0.3 & 8.3 & 6.3 & 5.9 & 3.8 & 3.8 & 4.5 & 13.5 \\
\hline Leptochloa filiformis & 3 & 1 & 0.1 & 1.0 & 3.0 & 1.5 & 1.8 & 0.5 & 1.3 & 3.8 \\
\hline Lindernia dubia & 4 & 1 & 0.1 & 1.3 & 4.0 & 1.5 & 2.5 & 0.6 & 1.5 & 4.5 \\
\hline Mollugo verticillata & 378 & 8 & 0.7 & 126.0 & 47.3 & 11.8 & 29.0 & 57.4 & 32.7 & 98.2 \\
\hline Murdannia nudiflora & 3 & 2 & 0.2 & 1.0 & 1.5 & 2.9 & 0.9 & 0.5 & 1.4 & 4.3 \\
\hline Paspalum maritimum & 24 & 7 & 0.6 & 8.0 & 3.4 & 10.3 & 2.1 & 3.6 & 5.3 & 16.0 \\
\hline Paspalum notatum & 6 & 4 & 0.3 & 2.0 & 1.5 & 5.9 & 0.9 & 0.9 & 2.6 & 7.7 \\
\hline Paspalum paniculatum & 57 & 2 & 0.2 & 19.0 & 28.5 & 2.9 & 17.5 & 8.7 & 9.7 & 29.1 \\
\hline Phyllanthus niruri & 3 & 3 & 0.3 & 1.0 & 1.0 & 4.4 & 0.6 & 0.5 & 1.8 & 5.5 \\
\hline Phyllanthus tenellus & 1 & 1 & 0.1 & 0.3 & 1.0 & 1.5 & 0.6 & 0.2 & 0.7 & 2.2 \\
\hline Pothomorphe umbellata & 7 & 2 & 0.2 & 2.3 & 3.5 & 2.9 & 2.1 & 1.1 & 2.1 & 6.2 \\
\hline Praxeliz pauciflora & 5 & 1 & 0.1 & 1.7 & 5.0 & 1.5 & 3.1 & 0.8 & 1.8 & 5.3 \\
\hline Rhychospora nervosa & 43 & 2 & 0.2 & 14.3 & 21.5 & 2.9 & 13.2 & 6.5 & 7.6 & 22.7 \\
\hline Spermacoce latifolia & 10 & 5 & 0.4 & 3.3 & 2.0 & 7.4 & 1.2 & 1.5 & 3.4 & 10.1 \\
\hline Spigelia anthelmia & 8 & 7 & 0.6 & 2.7 & 1.1 & 10.3 & 0.7 & 1.2 & 4.1 & 12.2 \\
\hline Sporobulus indicus & 1 & 1 & 0.1 & 0.3 & 1.0 & 1.5 & 0.6 & 0.2 & 0.7 & 2.2 \\
\hline Vernonia polyanthes & 1 & 1 & 0.1 & 0.3 & 1.0 & 1.5 & 0.6 & 0.2 & 0.7 & 2.2 \\
\hline Total & 658 & - & 5.7 & 219.3 & 163.2 & 100 & 100 & 100 & 100 & - \\
\hline
\end{tabular}

Cyperus esculentus and Kyllinga odorata (Cyperaceae) species are mainly herbaceous perennials, and their propagation occurs by seeds or rhizomes and subterranean tubers. A prolonged permanence of tubers in the soil makes it difficult to control these weeds, resulting in costly consequences and causing damage to the agricultural sector. The shade provided by the banana 
canopy promotes a reduction in the number of sprouts, tubers and dry matter production (KEELEY; THULLEN, 1978), which may be responsible for the low density of these plants in the area.

Table 6 - Values of the number of individuals (NI), number of squares (NQ), absolute frequency $(\mathrm{Fa})$, absolute density $(\mathrm{Da})$, absolute abundance $(\mathrm{Ab})$, relative frequency $(\mathrm{Fr})$, relative abundance (Ar), relative density (Dr), relative importance (Ir) and the importance value index (IVI) in area 4. Rio Branco-AC, 2014.

\begin{tabular}{|c|c|c|c|c|c|c|c|c|c|c|}
\hline Species & NI & NQ & $\mathrm{Fa}$ & $\begin{array}{l}\mathrm{Da} \\
\left(\mathrm{p} \mathrm{m}^{2}\right)\end{array}$ & $\mathrm{Ab}$ & Fr $(\%)$ & $\operatorname{Ar}(\%)$ & $\operatorname{Dr}(\%)$ & $\operatorname{Ir}(\%)$ & IVI \\
\hline Acalypha arvensis & 2 & 2 & 0.2 & 0.7 & 1.0 & 2.4 & 0.8 & 0.2 & 1.1 & 3.4 \\
\hline Alternanthera tenella & 1 & 1 & 0.1 & 0.3 & 1.0 & 1.2 & 0.8 & 0.1 & 0.7 & 2.1 \\
\hline Brachiaria brizantha & 5 & 1 & 0.1 & 1.7 & 5.0 & 1.2 & 3.8 & 0.6 & 1.9 & 5.6 \\
\hline Brachiaria decumbens & 1 & 1 & 0.1 & 0.3 & 1.0 & 1.2 & 0.8 & 0.1 & 0.7 & 2.1 \\
\hline Cecropia pachystachya & 5 & 2 & 0.2 & 1.7 & 2.5 & 2.4 & 1.9 & 0.6 & 1.6 & 4.9 \\
\hline Commelina benghalensis & 2 & 2 & 0.2 & 0.7 & 1.0 & 2.4 & 0.8 & 0.2 & 1.1 & 3.4 \\
\hline Cyperus esculentus & 7 & 5 & 0.4 & 2.3 & 1.4 & 6.1 & 1.1 & 0.8 & 2.7 & 8.0 \\
\hline Cyperus iria & 9 & 3 & 0.3 & 3.0 & 3.0 & 3.7 & 2.3 & 1.0 & 2.3 & 7.0 \\
\hline Desmodium barbatum & 1 & 1 & 0.1 & 0.3 & 1.0 & 1.2 & 0.8 & 0.1 & 0.7 & 2.1 \\
\hline Digitaria horizontalis & 7 & 3 & 0.3 & 2.3 & 2.3 & 3.7 & 1.8 & 0.8 & 2.1 & 6.2 \\
\hline Digitaria sanguinalis & 19 & 3 & 0.3 & 6.3 & 6.3 & 3.7 & 4.8 & 2.2 & 3.5 & 10.6 \\
\hline Fimbristylis dichotoma & 3 & 3 & 0.3 & 1.0 & 1.0 & 3.7 & 0.8 & 0.3 & 1.6 & 4.8 \\
\hline Kyllinga odorata & 19 & 5 & 0.4 & 6.3 & 3.8 & 6.1 & 2.9 & 2.2 & 3.7 & 11.2 \\
\hline Leptochloa filiformis & 40 & 4 & 0.3 & 13.3 & 10.0 & 4.9 & 7.6 & 4.6 & 5.7 & 17.1 \\
\hline Lindernia dubia & 4 & 2 & 0.2 & 1.3 & 2.0 & 2.4 & 1.5 & 0.5 & 1.5 & 4.4 \\
\hline Mollugo verticillata & 627 & 11 & 0.9 & 209.0 & 57.0 & 13.4 & 43.3 & 71.7 & 42.8 & 128.4 \\
\hline Paspalum conjugatum & 1 & 1 & 0.1 & 0.3 & 1.0 & 1.2 & 0.8 & 0.1 & 0.7 & 2.1 \\
\hline Paspalum maritimum & 40 & 5 & 0.4 & 13.3 & 8.0 & 6.1 & 6.1 & 4.6 & 5.6 & 16.7 \\
\hline Paspalum notatum & 1 & 1 & 0.1 & 0.3 & 1.0 & 1.2 & 0.8 & 0.1 & 0.7 & 2.1 \\
\hline Phyllanthus niruri & 9 & 2 & 0.2 & 3.0 & 4.5 & 2.4 & 3.4 & 1.0 & 2.3 & 6.9 \\
\hline Phyllanthus tenellus & 8 & 2 & 0.2 & 2.7 & 4.0 & 2.4 & 3.0 & 0.9 & 2.1 & 6.4 \\
\hline Praxeliz pauciflora & 39 & 6 & 0.5 & 13.0 & 6.5 & 7.3 & 4.9 & 4.5 & 5.6 & 16.7 \\
\hline Pueraria phaseoloides & 2 & 2 & 0.2 & 0.7 & 1.0 & 2.4 & 0.8 & 0.2 & 1.1 & 3.4 \\
\hline Spermacoce latifolia & 6 & 5 & 0.4 & 2.0 & 1.2 & 6.1 & 0.9 & 0.7 & 2.6 & 7.7 \\
\hline Spigelia anthelmia & 12 & 6 & 0.5 & 4.0 & 2.0 & 7.3 & 1.5 & 1.4 & 3.4 & 10.2 \\
\hline Talinum paniculatum & 4 & 2 & 0.2 & 1.3 & 2.0 & 2.4 & 1.5 & 0.5 & 1.5 & 4.4 \\
\hline Vernonia polyanthes & 1 & 1 & 0.1 & 0.3 & 1.0 & 1.2 & 0.8 & 0.1 & 0.7 & 2.1 \\
\hline Total & 875 & - & 6.8 & 291.7 & 131.6 & 100 & 100 & 100 & 100 & - \\
\hline
\end{tabular}

Although Spermacoce latifolia (oval-leaf false buttonweed) displayed high absolute and relative frequencies and tolerated a certain degree of shading, it was not one of the main weeds in 76 


\section{WEED INCIDENCE IN AN INTERCROPPING SYSTEM OF BANANA, TYPE PLANTAIN, CV.}

D'ANGOLA, WITH ASSAI PALM IN DIFFERENT ARRANGEMENTS

area 4 , with an absolute density of 2.0 plants $\mathrm{m}^{2}$, possibly due to an improvement in the fertility levels (LORENZI, 2008).

The use of banana plant remains is an important means of control, since it reduces weed density through light reduction or suppression, affecting weed germination and development when residues remain on the soil (OLIVEIRA; SOUZA, 2003). Consequently, the use of post-emergent herbicides can be rationalized, since some species are tolerant and require higher doses or herbicide combinations for control, such as Spermacoce latifolia and Commelina benghalensis.

The weed species with the highest absolute and relative frequencies in 4 × 2 × $2 \mathrm{~m}$ banana intercropped with $6 \times 3 \mathrm{~m}$ E. precatoria (area 5) were Paspalum maritimum, Spigelia anthelmia, Digitaria sanguinalis, Cyperus iria, Paspalum conjugatum, Fimbristylis dichotoma, and Praxelis pauciflora (Table 7).

It was observed that Digitaria sanguinalis had a higher phytosociological parameter for absolute density (55.0 plants per $\mathrm{m}^{2}$ ), absolute abundance $(33.0 \%)$, relative abundance $(24.1 \%)$, relative density (29.6\%), relative importance (20.2\%) and importance value index (60.6). Lima et al. (2012) and Gomes et al. (2010) demonstrated that this species is one of the main weeds found amid banana crops.

Table 8 shows that Mollugo verticillata, Fimbristylis dichotoma, Kylinga odorata and Phyllanthus niruri were the most frequent species in area 6 (4 × 2 × 2 m banana intercropped with $4 \times 2 \times 3 \mathrm{~m}$ E. precatoria palm), presenting higher absolute and relative frequencies.

In relation to the number of plants per $\mathrm{m}^{2}$, Mollugo verticillata and Paspalum notatum showed an absolute density of 60.3 and 24.0 plants per $\mathrm{m}^{2}$, relative density of $39.6 \%$ and $15.8 \%$, respectively, above the other results. Regarding absolute and relative abundance, relative importance and importance value index, one can observe that Digitaria sanguinalis, as well as Mollugo verticillata and Paspalum notatum stand out in this crop area.

Several phytosociological surveys carried out on banana crops and agroforestry intercroppings have identified many weed species belonging to different families (LIMA et al., 2012; GOMES et al., 2010; SILVA et al., 2013), which may be influenced by farming practices, soil management systems, location and time of survey, planting densities, among other factors. 
Table 7 - Values of the number of individuals (NI), number of squares (NQ), absolute frequency $(\mathrm{Fa})$, absolute density $(\mathrm{Da})$, absolute abundance $(\mathrm{Ab})$, relative frequency $(\mathrm{Fr})$, relative abundance (Ar), relative density (Dr), relative importance (Ir) and the importance value index (IVI) in area 5. Rio Branco-AC, 2014.

\begin{tabular}{|c|c|c|c|c|c|c|c|c|c|c|}
\hline Species & $\mathrm{NI}$ & $\mathrm{NQ}$ & $\mathrm{Fa}$ & $\begin{array}{l}\mathrm{Da} \\
\left(\mathrm{p} \mathrm{m}^{2}\right)\end{array}$ & $\mathrm{Ab}$ & $\operatorname{Fr}(\%)$ & $\operatorname{Ar}(\%)$ & $\operatorname{Dr}(\%)$ & $\operatorname{Ir}(\%)$ & IVI \\
\hline Acalypha arvensis & 1 & 1 & 0.1 & 0.3 & 1.0 & 1.4 & 0.7 & 0.2 & 0.8 & 2.3 \\
\hline Commelina benghalensis & 2 & 1 & 0.1 & 0.7 & 2.0 & 1.4 & 1.5 & 0.4 & 1.1 & 3.2 \\
\hline Corchorus olitorius & 2 & 1 & 0.1 & 0.7 & 2.0 & 1.4 & 1.5 & 0.4 & 1.1 & 3.2 \\
\hline Cyperus esculentus & 2 & 2 & 0.2 & 0.7 & 1.0 & 2.7 & 0.7 & 0.4 & 1.3 & 3.8 \\
\hline Cyperus iria & 27 & 5 & 0.4 & 9.0 & 5.4 & 6.8 & 3.9 & 4.8 & 5.2 & 15.6 \\
\hline Digitaria sanguinalis & 165 & 5 & 0.4 & 55.0 & 33.0 & 6.8 & 24.1 & 29.6 & 20.2 & 60.6 \\
\hline Eleusine indica & 37 & 2 & 0.2 & 12.3 & 18.5 & 2.7 & 13.5 & 6.6 & 7.6 & 22.9 \\
\hline Emilia fosbergii & 1 & 1 & 0.1 & 0.3 & 1.0 & 1.4 & 0.7 & 0.2 & 0.8 & 2.3 \\
\hline Fimbristylis dichotoma & 10 & 5 & 0.4 & 3.3 & 2.0 & 6.8 & 1.5 & 1.8 & 3.4 & 10.1 \\
\hline Kyllinga brevifolia & 9 & 3 & 0.3 & 3.0 & 3.0 & 4.1 & 2.2 & 1.6 & 2.6 & 7.9 \\
\hline Kyllinga odorata & 11 & 4 & 0.3 & 3.7 & 2.8 & 5.5 & 2.0 & 2.0 & 3.2 & 9.5 \\
\hline Leptochloa filiformis & 55 & 4 & 0.3 & 18.3 & 13.8 & 5.5 & 10.1 & 9.9 & 8.5 & 25.4 \\
\hline Mollugo verticillata & 60 & 4 & 0.3 & 20.0 & 15.0 & 5.5 & 11.0 & 10.8 & 9.1 & 27.2 \\
\hline Murdannia nudiflora & 7 & 4 & 0.3 & 2.3 & 1.8 & 5.5 & 1.3 & 1.3 & 2.7 & 8.0 \\
\hline Paspalum conjugatum & 25 & 5 & 0.4 & 8.3 & 5.0 & 6.8 & 3.7 & 4.5 & 5.0 & 15.0 \\
\hline Paspalum maritimum & 38 & 7 & 0.6 & 12.7 & 5.4 & 9.6 & 4.0 & 6.8 & 6.8 & 20.4 \\
\hline Paspalum paniculatum & 2 & 1 & 0.1 & 0.7 & 2.0 & 1.4 & 1.5 & 0.4 & 1.1 & 3.2 \\
\hline Phyllanthus niruri & 8 & 3 & 0.3 & 2.7 & 2.7 & 4.1 & 2.0 & 1.4 & 2.5 & 7.5 \\
\hline Praxeliz pauciflora & 44 & 5 & 0.4 & 14.7 & 8.8 & 6.8 & 6.4 & 7.9 & 7.1 & 21.2 \\
\hline Spermacoce latifolia & 3 & 3 & 0.3 & 1.0 & 1.0 & 4.1 & 0.7 & 0.5 & 1.8 & 5.4 \\
\hline Spigelia anthelmia & 46 & 6 & 0.5 & 15.3 & 7.7 & 8.2 & 5.6 & 8.3 & 7.4 & 22.1 \\
\hline Talinum paniculatum & 2 & 1 & 0.1 & 0.7 & 2.0 & 1.4 & 1.5 & 0.4 & 1.1 & 3.2 \\
\hline Total & 557 & - & 6.1 & 185.7 & 136.7 & 100 & 100 & 100 & 100 & - \\
\hline
\end{tabular}


Table 8 - Values of the number of individuals (NI), number of squares (NQ), absolute frequency $(\mathrm{Fa})$, absolute density $(\mathrm{Da})$, absolute abundance $(\mathrm{Ab})$, relative frequency $(\mathrm{Fr})$, relative abundance (Ar), relative density (Dr), relative importance (Ir) and the importance value index (IVI) in area 6. Rio Branco-AC, 2014.

\begin{tabular}{|c|c|c|c|c|c|c|c|c|c|c|}
\hline Species & $\mathrm{NI}$ & NQ & $\mathrm{Fa}$ & $\begin{array}{l}\mathrm{Da} \\
\left(\mathrm{p} \mathrm{m}^{2}\right)\end{array}$ & $\mathrm{Ab}$ & Fr $(\%)$ & $\operatorname{Ar}(\%)$ & $\operatorname{Dr}(\%)$ & $\operatorname{Ir}(\%)$ & IVI \\
\hline Mollugo verticillata & 181 & 8 & 0.7 & 60.3 & 22.6 & 14.0 & 15.4 & 39.6 & 23.0 & 69.0 \\
\hline Paspalum notatum & 72 & 3 & 0.3 & 24.0 & 24.0 & 5.3 & 16.3 & 15.8 & 12.4 & 37.3 \\
\hline Digitaria sanguinalis & 35 & 1 & 0.1 & 11.7 & 35.0 & 1.8 & 23.8 & 7.7 & 11.1 & 33.2 \\
\hline Paspalum maritimum & 34 & 4 & 0.3 & 11.3 & 8.5 & 7.0 & 5.8 & 7.4 & 6.7 & 20.2 \\
\hline Fimbristylis dichotoma & 31 & 5 & 0.4 & 10.3 & 6.2 & 8.8 & 4.2 & 6.8 & 6.6 & 19.8 \\
\hline Kyllinga odorata & 23 & 5 & 0.4 & 7.7 & 4.6 & 8.8 & 3.1 & 5.0 & 5.6 & 16.9 \\
\hline Cyperus iria & 20 & 3 & 0.3 & 6.7 & 6.7 & 5.3 & 4.5 & 4.4 & 4.7 & 14.2 \\
\hline Cyperus difusus & 11 & 1 & 0.1 & 3.7 & 11.0 & 1.8 & 7.5 & 2.4 & 3.9 & 11.6 \\
\hline Praxeliz pauciflora & 11 & 3 & 0.3 & 3.7 & 3.7 & 5.3 & 2.5 & 2.4 & 3.4 & 10.2 \\
\hline Phyllanthus niruri & 8 & 5 & 0.4 & 2.7 & 1.6 & 8.8 & 1.1 & 1.8 & 3.9 & 11.6 \\
\hline Commelina benghalensis & 7 & 1 & 0.1 & 2.3 & 7.0 & 1.8 & 4.8 & 1.5 & 2.7 & 8.0 \\
\hline Cyperus esculentus & 5 & 4 & 0.3 & 1.7 & 1.3 & 7.0 & 0.8 & 1.1 & 3.0 & 9.0 \\
\hline Murdannia nudiflora & 4 & 2 & 0.2 & 1.3 & 2.0 & 3.5 & 1.4 & 0.9 & 1.9 & 5.7 \\
\hline Paspalum conjugatum & 4 & 1 & 0.1 & 1.3 & 4.0 & 1.8 & 2.7 & 0.9 & 1.8 & 5.3 \\
\hline Spermacoce latifolia & 3 & 3 & 0.3 & 1.0 & 1.0 & 5.3 & 0.7 & 0.7 & 2.2 & 6.6 \\
\hline Phyllanthus tenellus & 1 & 1 & 0.1 & 0.3 & 1.0 & 1.8 & 0.7 & 0.2 & 0.9 & 2.7 \\
\hline Chamaecrista rotundifolia & 1 & 1 & 0.1 & 0.3 & 1.0 & 1.8 & 0.7 & 0.2 & 0.9 & 2.7 \\
\hline Spigelia anthelmia & 1 & 1 & 0.1 & 0.3 & 1.0 & 1.8 & 0.7 & 0.2 & 0.9 & 2.7 \\
\hline Eleusine indica & 1 & 1 & 0.1 & 0.3 & 1.0 & 1.8 & 0.7 & 0.2 & 0.9 & 2.7 \\
\hline Corchorus olitorius & 1 & 1 & 0.1 & 0.3 & 1.0 & 1.8 & 0.7 & 0.2 & 0.9 & 2.7 \\
\hline Ipomoea triloba & 1 & 1 & 0.1 & 0.3 & 1.0 & 1.8 & 0.7 & 0.2 & 0.9 & 2.7 \\
\hline Sporobulus indicus & 1 & 1 & 0.1 & 0.3 & 1.0 & 1.8 & 0.7 & 0.2 & 0.9 & 2.7 \\
\hline Cyperus flavus & 1 & 1 & 0.1 & 0.3 & 1.0 & 1.8 & 0.7 & 0.2 & 0.9 & 2.7 \\
\hline Total & 457 & - & 4.8 & 152.3 & 147.1 & 100 & 100 & 100 & 100 & - \\
\hline
\end{tabular}


In general, Mollugo verticillata stood out in all the assessed areas as the main weed in three areas (4, 5 and 6) regarding the phytosociological parameters (Tables 6, 7 and 8). The importance of this species in both intercropping and monoculture systems can be explained by its adaptation to carbon metabolism occurring in an intermediate form $\left(\mathrm{C}_{3}-\mathrm{C}_{4}\right)$, with $\mathrm{C}_{3}$ e $\mathrm{C}_{4}$ acids as the primary products of photosynthesis, the intermediate photorespiration and perivascular sheath containing chloroplasts, enabling a successful development in a shaded or sunny environment.

The E. precatoria palm monoculture favored a better weed development, resulting in a greater amount of dry matter, reaching a value of $2676.8 \mathrm{~kg} \mathrm{ha}^{-1}$ and, as a consequence, the percentage of soil cover was also higher, achieving 8.3 points on the grade scale (Tables 1 and 9).

Table 9 - Dry matter of the aerial part (MSPA) and grades related to the visual evaluation of the soil cover (NCS) in the E. precatoria palm intercropping with banana and monocultures. Rio Branco-AC, 2014.

\begin{tabular}{|c|c|c|}
\hline Treatments & $\operatorname{MSPA}\left(\mathrm{kg} \mathrm{ha}^{-1}\right)$ & NCS $(\%)$ \\
\hline E. precatoria palm monoculture (3 x $4 \mathrm{~m})$ & $2676.80 \mathrm{a}$ & $8.37 \mathrm{a}$ \\
\hline Banana monoculture ( 3 x $3 \mathrm{~m})$ & $555.87 \mathrm{~b}$ & $4.45 b$ \\
\hline Banana $(3 \times 2 \mathrm{~m})$ with $E$. precatoria palm $(3 \times 4 \mathrm{~m})$ & $379.13 b$ & $2.57 \mathrm{~b}$ \\
\hline Banana (3x $3 \mathrm{~m})$ with $E$. precatoria palm $(3 \times 4 \mathrm{~m})$ & $353.80 \mathrm{~b}$ & $3.63 b$ \\
\hline Banana (4 x $2 \times 2$ m) with E. precatoria palm $(6 \times 3 \mathrm{~m})$ & $272.33 b$ & $2.39 b$ \\
\hline Banana $(4 \times 2 \times 2$ m) with $E$. precatoria palm ( $4 \times 2 \times 3$ m) & $331.80 b$ & $2.39 b$ \\
\hline $\mathrm{CV}(\%)$ & 10.69 & 14.96 \\
\hline
\end{tabular}

Thus, the soil cover values ranged between 90 and 100\% with the presence of weeds above the extremely high incidence, in which monocotyledon species were favored by the high solar radiation, a consequence of the spacing and low height of the E. precatoria palms at the moment of the survey (LARCHER, 2004).

In areas with banana trees, both in monoculture and in an intercropping system with $E$. precatoria palm in different planting arrangements, there was no statistical difference in the production of dry mass and visual evaluation scores (Tables 1 and 9). The grades ranged from 2.39 to 4.45 ( 1 to $12.5 \%$ of the soil cover), indicating that the shading imposed by banana leaves promoted an efficient reduction in the soil cover levels, and was also responsible for the decrease of species diversity and for the reduction in the dry mass of the aerial part of the weeds. Such 80 
influence on the weed community occurred due to the low-intensity light in relation to the $E$. precatoria palm monoculture.

The similarity index indicated similarities in the weed communities found in E. precatoria palm and banana monocultures, as well as in intercropping systems with different planting arrangements, which achieved a value above $60 \%$ (Table 10).

Table 10 - Similarity index values (IS\%) obtained from the survey carried out on the floristic communities of different areas under banana intercropping with E. precatoria palm and monocultures in different planting densities.

\begin{tabular}{llllll}
\hline IS\% & Area 1 & Area 2 & Area 3 & Area 4 & Area 5 \\
\hline Area 2 & 67.74 & & & & \\
Area 3 & 65.57 & 80.00 & & & \\
Area 4 & 76.67 & 70.37 & 71.70 & & \\
Area 5 & 64.29 & 72.00 & 73.47 & 66.67 & \\
Area 6 & 63.16 & 66.67 & 64.00 & 61.22 & 75.56 \\
\hline
\end{tabular}

The major similarities were found between the species of area 2 and area $3(80.0 \%)$, area 1 and area $4(76.67 \%)$, area 5 and area $6(75.56 \%)$, which can be explained in part by the management practices adopted, the proximity between areas and the same type of soil (CARVALHO; PITELLI, 1992).

Comparing the banana monoculture (area 2) with the intercropping system in different planting densities, it was observed that there was less similarity between the species in area 6, with a similarity index of $66.67 \%$, due to the greater density of E. precatoria palms and banana trees. Likewise, Souza et al. (2003) found a lower similarity index when comparing peach-palm and cupuaçu monocultures with an agroforestry system of these crops in the Amazonian environmental conditions. Contrasting with the present study, Gomes et al. (2010) observed a low similarity between weed species when comparing drained floodplain and banana dry farming areas (35.70\%).

\section{CONCLUSIONS}

In general, Mollugo verticillata is one of the main species found in E. precatoria palm and banana monocultures and in intercropping systems with different planting arrangements. 
The E. precatoria palm monoculture shows a greater diversity of weed species and individuals, dry mass of the aerial part and provides a considerable soil cover.

Areas with banana monoculture or under a banana intercropping with E. precatoria palm in different planting arrangements display the same amount of dry matter of the aerial part and similar soil cover levels.

\section{REFERENCES}

AlbuQuerque, J. A. A.; EVAngElista, M. O.; MATES, A. P. K.; ALVES, J. M. A.; OLIVEIRA, N. T.; SEDIYAMA, T.; SILVA, A. A. 2014. Occurrence of weeds in Cassava savana plantations in Roraima. Planta Daninha, Viçosa, MG, v. 32, n. 1, p. 91-98.

BARRO, R. S.; VARELLA, A. C.; BANGEL, F. V.; SAIBRO, J. C.; MEDEIROS, R. B.; RADIN, B. 2010. Screening native C4 pasture genotypes for shade tolerance in Southern Brazil. In: AUSTRALIAN SOCIETY OF AGRONOMY CONFERENCE, 15., Lincoln, Nova Zelândia. Proceedings... Lincoln: Australian Society of Agronomy, 2010. Available in: <http://www.regional.org.au/au/asa/2010/crop-production/intercrops/6969_varellaac. htm>. Access em: 6 july 2015.

BELALCÁZAR CARVAJAL, S.; SALAZAR M., C. A.; CAYÓN S., G.; LOZADA Z. J. E.; CASTILLO, L. E.; VALENCIA M., J. A. 1991. Manejo de Plantaciones. In: El cultivo del plátano en el tropico. Colômbia: INIBAP/ICA/CDCT/ CIID. p. 149-242. (Manual de Asistencia tecnica, 50).

CARVAlHO, S. L.; PITELli, R. A. 1992. Comportamento e análise fitossociológica das principais espécies de plantas daninhas de pastagens da região de Selvia (MS). Planta Daninha, Viçosa, MG, v.10, p. 25-32.

DEUBER, R. 1992. Ciência das plantas daninhas: fundamentos. Jaboticabal: FUNEP, 431 p.

ERASMO, E. A. L.; PINHEIRO, L. L. A.; COSTA, N. V. 2004. Levantamento fitossociológico das comunidades de plantas infestantes em áreas de produção de arroz irrigado cultivado sob diferentes sistemas de manejo. Planta Daninha, Viçosa, MG, v. 22, n. 2, p. 195-201.

GOMES, G. L. G. C.; IBRAHIM, F. N.; MACEDO, G. L.; NOBREGA, L. P.; ALVES, E. 2010. Cadastramento fitossociológico de plantas daninhas na bananicultura. Planta Daninha, Viçosa, MG, v. 28, n. 1, p. 61-68.

IBGE, 2019. Instituto Brasileiro de Geografia e Estatística. Lavouras permanentes. Available in: http://www.ibge.gov.br/home/estatistica/ economia/pam/default_perm_xls.shtm. Access in: 17 Abril, 2019.

KEELEY, P. E.; THULLEN, R. J. 1978. Light requirements of yellow nutsedge (Cyperus esculentus) and light interception by crops. Weed Science, New York, p. 10-16.

KISSMANN, K. G.; GROTH, D. 1997. Plantas infestantes e nocivas. São Paulo: BASF Brasileira, v. 2, 798 p.

LANZA, T.R.; MACHADO, A.F.L.; MARTELLETO, L.A.P. 2017. Effect of planting densities of "BRS Princess" banana tree in the suppression of weeds. Planta daninha, Viçosa, v.35, e017162958.

LARCHER, W. 2004. Ecofisiologia vegetal. São Carlos, SP: RiMa. 531 p. 
LIMA, L. K. S.; BARBOSA, A. J. S.; SILVA, R. T. L. da; ARAÚJO, R. da C. 2012. Distribuição fitossociológica da comunidade de plantas espontâneas na bananicultura. Revista Verde, Mossoró, v. 7, n. 4, p. 59-68.

LORENZI, H. 2008. 4 ed. Plantas daninhas do Brasil: terrestres, aquáticas, parasíticas e tóxicas. Nova Odessa: Instituto Plantarum.

MOURA FILHO, E. R.; MACEDO, L. P. M.; SILVA, A. R. S. 2015. Levantamento fitossociológico de plantas daninhas em cultivo de banana irrigada. Holos, v. 2, p. 92-97.

OLIVEIRA, A. R.; FREITAS, S. P. 2008. Levantamento fitossociológico de plantas daninhas em áreas de produção de cana-de-açúcar. Planta Daninha, Viçosa, MG, v. 26, n. 1, p. 33-46.

OLIVEIRA, C. A. P. de; SOUZA, C. M. de. 2003. Influência da cobertura morta na umidade, incidência de plantas daninhas e de broca-do-rizoma (Cosmopolites sordidus) em um pomar de bananeiras (Musa spp.). Revista Brasileira de Fruticultura, Jaboticabal, v. 25, n. 2, p. 345-347.

OUMA, G.; JERUTO, P. 2010. Sustainable horticultural crop production through intercropping: The case of fruits and vegetable crops: A review. Agriculture and Biology Journal of North America, vol. 1, p. 1098-1105.

SARMENTO, H. G. S.; CAMPOS FILHO, J. M.; ASPIAZÚ, I.; RODRIGUES, T. M.; FERREIRA, E. A. 2015. Levantamento fitossociológico de plantas daninhas em áreas de bananicultura no Vale do Rio Gorutuba, norte de Minas Gerais. Revista Agro@mbiente Online, Boa Vista-RR, v. 9, n. 3, p. 308-316.

SCHREINER, H. G. 1987 Tolerância de quatro gramíneas forrageiras a diferentes graus de sombreamento. Boletim de Pesquisa Florestal, Colombo, n. 15, p.61-72, Dez. Available in: < http://www.cnpf.embrapa.br/pesquisa/safs/bolet15a.pdf>. Access in: 6 July. 2015.

SILVA, V. de C.; PERDONÁ, M. J.; SORATTO, R. P.; NEGRISOLI, E. 2013. Ocorrência de plantas daninhas em cultivo consorciado de café e nogueira-macadâmia. Pesquisa Agropecuária Tropical, Goiânia, v. 43, n. 4, p. 441-449.

SOUZA, L. S. A.; SILVA, J. F.; SOUZA, M. D. B. 2003. Composição florística de plantas daninhas em agrossistemas de cupuaçuzeiro (Theobroma grandiflorum) e pupunheira (Bactris gasipaes). Planta Daninha, Viçosa, MG, v. 21, n. 2, p. 249-255.

VIEIRA, T. A.; ROSA, L. dos S.; VASCONCELOS, P. C. S.; SANTOS, M. M. dos S.; MODESTO, R. da S. 2007. Sistemas agroflorestais em áreas de agricultores familiares em Igarapé-Açu, Pará: caracterização florística, implantação e manejo. Acta Amazonica, Manaus, v. 37 , n. 4 , p. 549-558. 